\title{
Revisiting the crystal structure and magneto-elastic coupling in MnZnSb
}

\author{
K. Routledge ${ }^{1}$, P.A.E Murgatroyd ${ }^{1}$, J.B. Claridge ${ }^{2}$, S.N. Savvin ${ }^{3}$ and J. Alaria ${ }^{1}$ \\ ${ }^{1}$ Department of Physics, University of Liverpool, Oxford Street, Liverpool L69 7ZE, UK, \\ ${ }^{2}$ Department of Chemistry, University of Liverpool, Crown Street, Liverpool L69 7ZD, UK, \\ ${ }^{3}$ Institut Laue-Lengevin, 71 Avenue des Martyrs, Grenoble 38000, France.
}

\section{K.Routledge@liverpool.ac.uk}

Magneto-caloric materials offer the possibility to design environmentally friendlier thermal management devices compared to the widely used gas-based systems [1]. These materials exhibit a change in entropy $\left(\Delta \mathrm{S}_{\mathrm{M}}\right)$ or a temperature change $\left(\Delta \mathrm{T}_{\mathrm{ad}}\right)$ when subjected to a magnetic field under isothermal or adiabatic conditions, respectively. The magnitude of change is largest about the materials Curie temperature $\left(\mathrm{T}_{\mathrm{c}}\right)$ due to the order-disorder phase transition of the magnetic moments within the system. A suitable material must present a large magneto-caloric effect over a broad temperature span together with suitable secondary application parameters such as low heat capacity and high thermal conductivity. $\mathrm{MnZnSb}$ is derived from the $\mathrm{PbFCl}$ structure (in which the Mn sites are arranged within two-dimensional square nets), resulting in a pseudo 2D itinerant ferromagnetism which orders just above room temperature.

The first structural study reports that $\mathrm{MnZnSb}$ crystallises in the same anti-PbFCl-type structure as $\mathrm{Mn}_{2} \mathrm{Sb}$, with the tetragonal space group P4/nmm [2]. However, results from our experiments suggests that the true structure is more complex than this and has some aperiodic nature. Electron diffraction, in-house X-ray diffraction (Fig. 1(b)) and D20 neutron diffraction (Fig. 1(c)) data on powder samples all suggest that there is a small distortion of the tetragonal cell to a triclinic subgroup cell. As well as this, there appears to be incommensurate modulations in atomic positions and possibly Mn-Zn occupation (which is only seen in the neutron data). In the variable temperature neutron diffraction, we have also uncovered an unreported structural transition at $\sim 130 \mathrm{~K}$.

We have investigated the magneto-caloric properties of $\mathrm{MnZnSb}$ [3] using a combination of computational and experimental methods, including samples in which some $\mathrm{Mn}$ is substituted with $\mathrm{Fe}$ and $\mathrm{Cr}$. Scaling analysis of the magnetic properties determines that they are second order phase transition ferromagnets and neutron diffraction has determined that the magnetic entropy change (Fig. 1(a)) is driven by the coupling of magneto-elastic strain in the square net through the magnetic transition. The primary and secondary application related properties have been measured experimentally, and the $c / a$ parameter is identified as an accurate proxy to control the magnetic transition. Chemical substitution on the square net affords tuning of the Curie temperature over a broad temperature span between 252 and $322 \mathrm{~K}$.

(a)

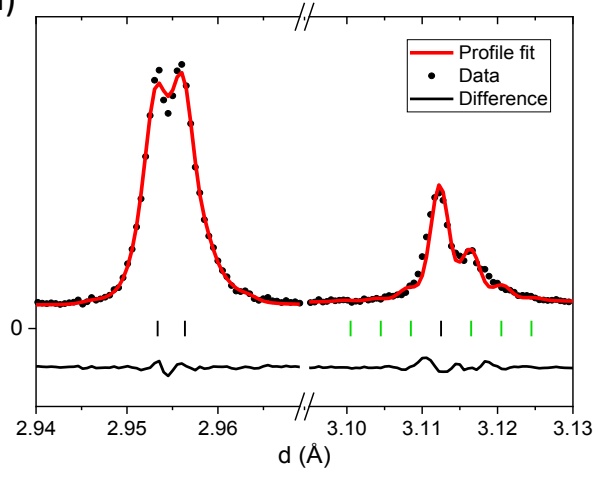

(b)

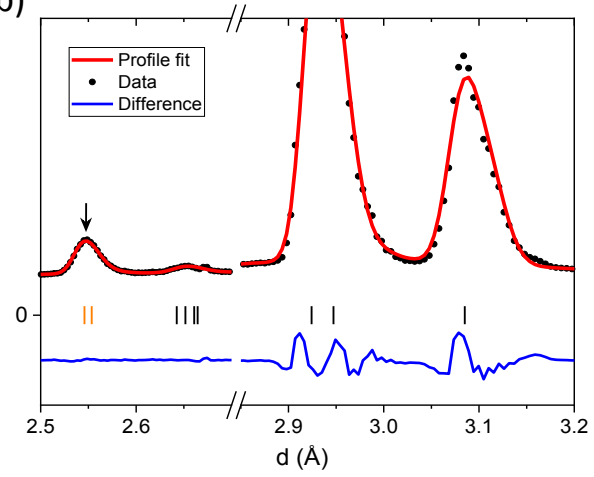

(c)

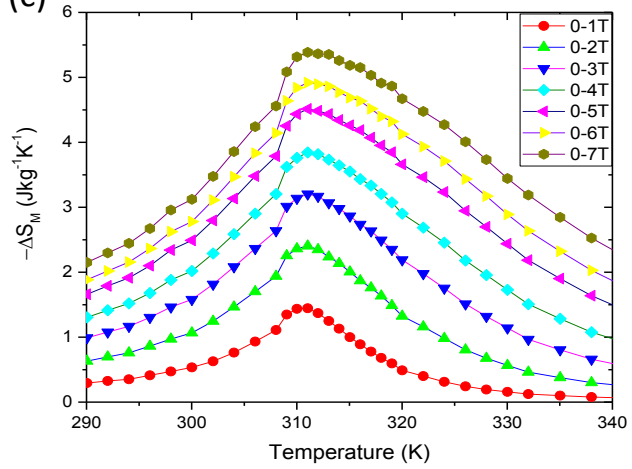

Figure 1. (a) RT Powder XRD data with P-1 $(\alpha \beta \gamma) 0$ model. The $2.955 \AA$ peak is split by making $\gamma \neq 90^{\circ}$. Positional satellite peak(s) around the 3.11 $\AA$ peak are fit with $\mathbf{q}=(0.00188(2), 0,0.00161(2))$, indicated with green ticks. (b) Neutron D20 data at 320K, where a suspected Mn-Zn occupation satellite $(2.55 \AA)$ is visible. Fitted in a P-1 $(\alpha \beta \gamma) 0$ model, where $\mathbf{q}=(0,0.3218(2), 0.3587(3))$ is fitted to the occupation peaks, indicated with orange ticks. (c) Magnetic entropy change $\left(\mathrm{Jkg}^{-1} \mathrm{~K}^{-1}\right)$ vs temperature $(\mathrm{K})$ for $\mathrm{MnZnSb}$ with magnetic field changes ranging from 0-1T to 0-7T.

[1] Franco, V. et al. (2018). Prog. Mater. Sci., 93, pp. 112-232, 4.

[2] Johnson, V. \& Jeitschko, W. (1977). J. Solid State Chem, 22, pp. 71-75.

[3] Murgatroyd, P.A.E. et. al. (2021). Adv. Funct. Mater., 31, 2100108.

Keywords: magnetocalorics; chemical control; magnetic materials; incommensurate

Acta Cryst. (2021), A77, C638 